\title{
A NOTE OF THE UNION-CLOSED SETS CONJECTURE
}

\author{
R. M. NORTON and D. G. SARVATE
}

(Received 28 March 1991; revised 30 September 1991)

\author{
Communicated by L. Caccetta
}

\begin{abstract}
Let $\mathscr{A}=\left\{A_{1}, \ldots, A_{n}\right\}$ be a union-closed set. This note establishes a property which must be possessed by any smallest counterexample to the Union-Closed Sets Conjecture. Specifically, a counterexample to the conjecture with minimal $n$ has at least three distinct elements, each of which appears in exactly $(n-1) / 2$ of the $A_{j}^{\prime} s$.

1991 Mathematics subject classification (Amer. Math. Soc.): 05 A 99.
\end{abstract}

\section{Introduction}

A union-closed set is defined as a nonempty finite collection of distinct nonempty finite sets closed under union. The following conjecture is referred to as the Union-Closed Sets Conjecture:

CONJECTURE. Let $\mathscr{A}=\left\{A_{1}, \ldots, A_{n}\right\}$ be a union-closed set. Then there exists an element which belongs to at least $\lceil n / 2\rceil$ of the $A_{j}^{\prime} s$, where

$$
\lceil n / 2\rceil= \begin{cases}n / 2 & \text { if } n \text { is even } \\ (n+1) / 2 & \text { if } n \text { is odd }\end{cases}
$$

For some historical remarks see [6]. The origin of the conjecture should be attributed to a problem of Peter Frankl (see [2, p. 525] and [5, pp. 161 and 186]).

(C) 1993 Australian Mathematical Society 0263-6115/93 \$A2.00+0.00 
Frankl's problem was stated so as to include the empty set in $\mathscr{A}$. In a letter to Fred Galvin, Frankl traces his posing of the problem to December, 1979, and mentions that in early 1980 he relayed the problem to Ron Graham, whom he credits with giving the conjecture some publicity. Sarvate and Renaud [3, 4] have given some bounds on the size of te smallest set in a counterexample to the conjecture, proved that the conjecture is true when $m \leq 18$, and given some other results. The purpose of this note is to give a necessary property for a counterexample of minimal $n$.

\section{A necessary property of a smallest counterexample}

Let $\mathscr{A}=\left\{A_{1}, \ldots, A_{n}\right\}$ be a union-closed set. Assume for convenience that $\left|A_{1}\right| \leq\left|A_{j}\right|$ for all $j$. Let $N$ be the minimum value of $n$ taken over all counterexamples to the union-closed sets conjecture. If a counterexample to the conjecture exists then $N$ is odd (see [3, Theorem 1] and the following theorem holds.

THEOREM. Let $\mathscr{A}=\left\{A_{1}, \ldots, A_{n}\right\}$ be a counterexample to the conjecture with $n$ minimal. Then there are at least three distinct elements, each of which appears in exactly $(n-1) / 2$ of the $A_{j}^{\prime} s$.

PROOF. Let $n=2 t+1$ and $\mathscr{B}=\left\{A_{2}, A_{3}, \ldots, A_{n}\right\}$. Now $\mathscr{B}$ is union-closed. Therefore there exists an element $x$ in $t$ sets of $\mathscr{B}$. Let $A_{x}$ be a smallest set containing $x$ in $\mathscr{B}$. Note that $x$ is not in $A_{1}$. Consider $\mathscr{C}=\mathscr{B}-\left\{A_{x}\right\}$ Now $\mathscr{C}$ is union-closed, so there exists an element $y(y \neq x)$ in $t$ sets of $\mathscr{C}$, hence in $t$ sets of $\mathscr{A}$. Let $A_{y}$ be a smallest set in $\mathscr{A}$ containing $y$. Certainly $A_{x}$ and $A_{y}$ are distinct sets. Let $\mathscr{D}=\left\{A_{1}, \ldots, A_{n}\right\}-\left\{A_{x}, A_{y}\right\}$. To see that $\mathscr{D}$ is union-closed, suppose to the contrary that $A_{i} \cup A_{j}$ is not in $\mathscr{D}$ for some $A_{i}, A_{j}$ in $\mathscr{D}$. This implies that $A_{i} \cup A_{j}=A_{x}$ or $A_{i} \cup A_{j}=A_{y}$. If $A_{i} \cup A_{j}=A_{x}$ then either $A_{i}$ or $A_{j}$ contains $x$. But since $A_{i}$ and $A_{j}$ are in $\mathscr{D}$, neither $A_{i}$ nor $A_{j}$ can equal $A_{x}$. So by the minimal cardinality assumption about $A_{x}, A_{i} \cup A_{j}$ cannot equal $A_{x}$. Similarly $A_{j} \cup A_{j} \neq A_{y}$. So $\mathscr{D}$ is union-closed, and there exists an element $z$ in $t$ sets of $\mathscr{D}$. Now $z \neq y$ and $z \neq x$, because otherwise $z$ is in $t+1$ sets of $\mathscr{A}$. Hence there are at least three elements each of which is in exactly $(n-1) / 2$ sets of $\mathscr{A}$. 


\section{References}

[1] 'A much travelled conjecture', Austral. Math. Soc. Gaz. 14 (1987), 63.

[2] (ed. I. Rival), Graphs and Order, (proceedings of the NATO advanced study institute on graphs and order) (Reidel, Dordrecht, 1984).

[3] D. G. Sarvate and J.-C. Renaud, 'On the union-closed sets conjecture', Ars Combin. 27 (1989), 149-154.

[4] _ _ 'Improved bounds for the union-closed sets conjecture', Ars Combin. 29 (1990), 181-185.

[5] R. P. Stanley, Enumerative Combinatorics (Wadsworth, Monterey, 1986).

[6] P. Winkler, 'Union-closed sets conjecture', Austral. Math. Soc. Gaz. 14 (1987), 99.

College of Charleston

Charleston, SC 29424

USA
College of Charleston

Charleston, SC 29424

USA 